\title{
The tacnode Riemann-Hilbert problem
}

\author{
Arno Kuijlaars \\ Department of Mathematics, KU Leuven \\ Celestijnenlaan 200 B bus 2400 \\ 3001 Leuven, Belgium \\ e-mail: arno.kuijlaars@wis.kuleuven.be \\ Published in \\ Constructive Approximation 39 (2014), 197-222. \\ DOI 10.1007/s00365-013-9225-Z
}

\begin{abstract}
The tacnode Riemann-Hilbert problem is a $4 \times 4$ matrix valued $\mathrm{RH}$ problem that appears in the description of the local behavior of two touching groups of non-intersecting Brownian motions. The same $\mathrm{RH}$ problem was also found by Duits and Geudens to describe a new critical regime in the two-matrix model.

Delvaux gave integral representations for some of the entries of the $4 \times 4$ matrix. We complement this work by presenting integral representations for all of the entries. As a consequence we give an explicit formula for the Duits-Geudens critical kernel.
\end{abstract}

Keywords Riemann-Hilbert problem, tacnode, integral representation, Painlevé II equation, Hastings-McLeod solution, two-matrix model

Mathematics Subject Classification Primary 30E20, 34M50, Secondary 15B52, 33E17, 34M55, 60J65

\section{Introduction}

The tacnode Riemann-Hilbert $(\mathrm{RH})$ problem is a $4 \times 4$ matrix-valued $\mathrm{RH}$ problem that first appeared in the asymptotic analysis of two touching groups of nonintersecting Brownian motions, a so-called tacnode. The positions of the non-intersecting Brownian motions are a determinantal point 
process that in a double scaling limit around the tacnode leads to the tacnode process. The one-time correlation functions of the tacnode process were expressed in terms of the tacnode $\mathrm{RH}$ problem in [12]. The tacnode $\mathrm{RH}$ problem is related to the Hastings-McLeod solution of the Painlevé II equation as was also discussed in [12].

The tacnode problem was also analyzed in $[2,17,23]$ using different techniques. In these papers the tacnode kernel and its multi-time extension are expressed in terms of integrals with resolvents of Airy integral operators acting on a half-line. These expressions are very different from the $\mathrm{RH}$ formulation.

Recently, Delvaux [10] made the connection between the two sets of formulas by presenting integral representations for some of the entries of the solution of the tacnode $\mathrm{RH}$ problem. These entries are exactly the ones that play a role for the tacnode kernel in [12]. With these explicit formulas Delvaux could make the connection between the formulas in [12] and the ones by Ferrari and Vetö [17] for the asymmetric tacnode. The paper [10] was inspired by the paper [6] by Baik, Liechty, and Schehr, where a connection between different sets of formulas for the maximal height and position of the Airy $_{2}$ process was made.

The aim of this paper is to complement the work of [10] by providing integral representations for all the entries of the tacnode $\mathrm{RH}$ problem. Some of these remaining entries appear in the description of a critical kernel appearing in the two-matrix model as shown by Duits and Geudens [13]. We therefore find explicit integral formulas for the Duits-Geudens critical kernel.

In section 2 we recall the tacnode $\mathrm{RH}$ problem with some of its properties, and in particular the connection with the Hastings-McLeod solution of Painlevé II. The main results of this paper are stated in Theorems 2.5, 2.8 and 2.9 below. We compare the solution of the tacnode $\mathrm{RH}$ problem with the explicit solution of the usual $2 \times 2$ matrix-valued $\mathrm{RH}$ problem for the Hastings-McLeod solution in section 2.6.

The proofs of Theorems 2.5 and 2.8 are in section 3. A key role is played by Lemma 3.2 that describes solutions to a certain ODE system (3.2). The proof of this lemma follows along the lines of certain proofs in [10]. We give full details about the calculations in section 5 . Following [10] we briefly mention the tacnode kernel in section 4.1. The implication of Theorem 2.8 for the Duits-Geudens critical kernel is given in Theorem 2.9. This theorem is proved in section 4 .

A variation of the tacnode $\mathrm{RH}$ problem for the hard-edge tacnode and the chiral two-matrix model appears in $[9,11]$. It may be possible that explicit integral representations for the solution of these $\mathrm{RH}$ problems can 


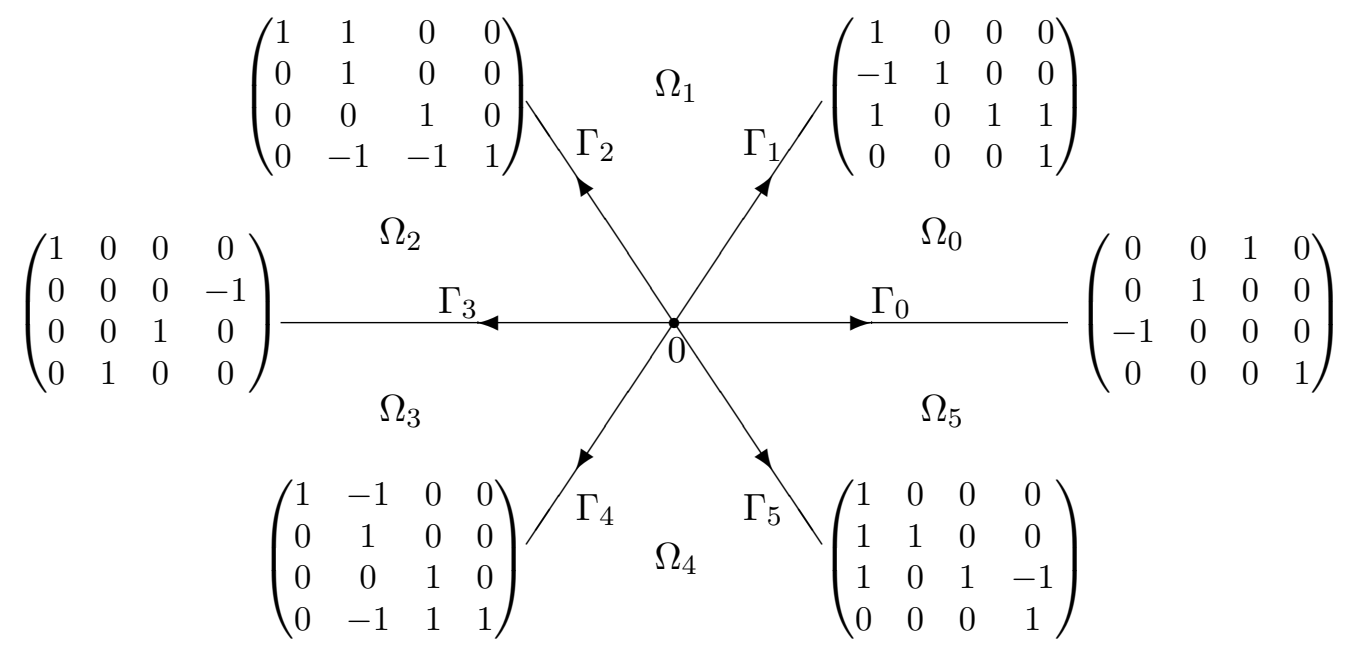

Figure 1: The figure shows the jump contours $\Gamma_{k}$ in the complex plane and the corresponding jump matrices $J_{k}, k=0, \ldots, 5$, in the tacnode $\mathrm{RH}$ problem. $\Omega_{k}$ is the sector bounded by the two rays $\Gamma_{k}$ and $\Gamma_{k+1}$.

be found as well. Other recent contributions $[1,3,7,20]$ discuss further connections and properties of the tacnode process.

\section{Statement of results}

\subsection{The tacnode RH problem}

The tacnode $\mathrm{RH}$ problem asks for a $4 \times 4$ matrix-valued function

$$
M: \mathbb{C} \backslash \Gamma_{M} \rightarrow \mathbb{C}^{4 \times 4}
$$

which is defined and analytic outside a set $\Gamma_{M}$ which is a union of six rays

$$
\Gamma_{M}=\bigcup_{k=0}^{5} \Gamma_{k}, \quad \Gamma_{k}=\left\{z \mid \arg z=\frac{\pi}{3} k\right\},
$$

as shown in Figure 1. Each ray is oriented from the origin to infinity. The orientation induces \pm -sides on each ray, where the + -side is on the left and 
the --side is on the right as one traverses the ray according to its orientation. We ask that $M$ has continuous boundary values $M_{ \pm}$on each of the rays that satisfy the jump condition

$$
M_{+}=M_{-} J_{k} \quad \text { on } \Gamma_{k} \text { for } k=0,1, \ldots, 5,
$$

where the jump matrix $J_{k}$ on $\Gamma_{k}$ is also shown in Figure 1.

The RH problem depends on a number of parameters $r_{1}, r_{2}, s_{1}, s_{2}, \tau$ that appear in the asymptotic condition for $M$ via two functions

$$
\begin{array}{ll}
\theta_{1}(z)=\frac{2}{3} r_{1}(-z)^{3 / 2}+2 s_{1}(-z)^{1 / 2}, & z \in \mathbb{C} \backslash[0, \infty), \\
\theta_{2}(z)=\frac{2}{3} r_{2} z^{3 / 2}+2 s_{2} z^{1 / 2}, & z \in \mathbb{C} \backslash(-\infty, 0],
\end{array}
$$

where we use principal branches for the fractional exponents. The asymptotic condition is

$$
\begin{aligned}
& M(z)=\left(I+\frac{M^{(1)}}{z}+O\left(z^{-2}\right)\right) \operatorname{diag}\left((-z)^{-1 / 4}, z^{-1 / 4},(-z)^{1 / 4}, z^{1 / 4}\right) \\
& \times \frac{1}{\sqrt{2}}\left(\begin{array}{cccc}
1 & 0 & -i & 0 \\
0 & 1 & 0 & i \\
-i & 0 & 1 & 0 \\
0 & i & 0 & 1
\end{array}\right) \operatorname{diag}\left(e^{-\theta_{1}(z)+\tau z}, e^{-\theta_{2}(z)-\tau z}, e^{\theta_{1}(z)+\tau z}, e^{\theta_{2}(z)-\tau z}\right) .
\end{aligned}
$$

The residue matrix $M^{(1)}$ is independent of $z$ but depends on the parameters.

Remark 2.1. In the papers $[12,13]$ the tacnode $\mathrm{RH}$ problem was formulated on a union of ten rays. Here we choose to combine the two jumps in each of the open quadrants which reduces the number of rays by four. It is easy to see that the two $\mathrm{RH}$ problems are equivalent.

Proposition 2.2. Suppose that the parameters $r_{1}, r_{2}, s_{1}, s_{2}, \tau$ are real with $r_{1}>0$ and $r_{2}>0$. Then the RH problem (2.2)-(2.4) has a unique solution.

Proposition 2.2 was proved in the case $\tau=0$ by Delvaux, Kuijlaars, and Zhang [12], and in the case $r_{1}=r_{2}=1, s_{1}=s_{2}$ with general $\tau$ by Duits and Geudens [13]. The proof in [13] extends to the general case as noted by Delvaux [10]. 


\subsection{The Painlevé II connection}

The tacnode $\mathrm{RH}$ problem is related to the Hastings-McLeod solution of the Painlevé II equation, as was noted in the cited papers $[10,12,13]$. The Painlevé II equation is

$$
q^{\prime \prime}=t q+2 q^{3}
$$

and the Hastings-McLeod solution is the unique solution of (2.5) that satisfies

$$
q(t)=\operatorname{Ai}(t)(1+o(1)) \quad \text { as } t \rightarrow+\infty
$$

where Ai denotes the usual Airy function. We also need the related function

$$
u=q^{\prime 2}-t q^{2}-q^{4},
$$

which satisfies $u^{\prime}=-q^{2}$.

Proposition 2.3. The solution $M$ of the tacnode $R H$ problem satisfies a differential equation

$$
\frac{\partial}{\partial z} M=U M
$$

with a matrix $U$ that is explicitly given in terms of the Hastings-McLeod solution $q$ of Painlevé II and u from (2.6) as follows:

$$
\begin{aligned}
& U= \\
& \left(\begin{array}{cccc}
\tau-s_{1}^{2}+\frac{u}{C} & \frac{\sqrt{r_{2}} q}{\gamma \sqrt{r_{1} C}} & i r_{1} & 0 \\
-\gamma \frac{\sqrt{r_{1}} q}{\sqrt{r_{2} C}} & -\tau+s_{2}^{2}-\frac{u}{C} & 0 & i r_{2} \\
i\left(r_{1} z-2 s_{1}+\frac{s_{1}^{4}}{r_{1}}-\frac{2 s_{1}^{2} u}{r_{1} C}+\frac{u^{2}-q^{2}}{r_{1} C^{2}}\right) & \frac{i}{\gamma}\left(\sqrt{r_{1} r_{2}} C\left(q^{\prime}+u q\right)-\frac{r_{1}^{2} s_{2}^{2}+r_{2}^{2} s_{1}^{2}}{\left(r_{1} r_{2}\right)^{3 / 2}} \frac{q}{C}\right) & \tau+s_{1}^{2}-\frac{u}{C} & \frac{\sqrt{r_{1}} q}{\gamma \sqrt{r_{2} C}} \\
i \gamma\left(\sqrt{r_{1} r_{2}} C\left(q^{\prime}+u q\right)-\frac{r_{1}^{2} s_{2}^{2}+r_{2}^{2} s_{1}^{2}}{\left(r_{1} r_{2}\right)^{3 / 2}} \frac{q}{C}\right) & i\left(-r_{2} z-2 s_{2}+\frac{s_{2}^{4}}{r_{2}}-\frac{2 s_{2}^{2} u}{r_{2} C}+\frac{u^{2}-q^{2}}{r_{2} C^{2}}\right) & -\gamma \frac{\sqrt{r_{2}} q}{\sqrt{r_{1} C}} & -\tau-s_{2}^{2}+\frac{u}{C}
\end{array}\right)
\end{aligned}
$$

Here $r_{1}, r_{2}, s_{1}, s_{2}, \tau$ are the parameters in the problem,

$$
\begin{aligned}
C & =\left(r_{1}^{-2}+r_{2}^{-2}\right)^{1 / 3}, \\
\gamma & =\exp \left(\frac{8}{3} \frac{r_{1}^{2}-r_{2}^{2}}{\left(r_{1}^{2}+r_{2}^{2}\right)^{2}} \tau^{3}-4 \frac{r_{1} s_{1}-r_{2} s_{2}}{r_{1}^{2}+r_{2}^{2}} \tau\right),
\end{aligned}
$$

and the Painlevé functions $q, q^{\prime}$, and $u$ that appear in (2.8) are evaluated in

$$
t=\frac{2}{C}\left(\frac{s_{1}}{r_{1}}+\frac{s_{2}}{r_{2}}-\frac{2 \tau^{2}}{r_{1}^{2}+r_{2}^{2}}\right)
$$


Proof. See Delvaux [10]. Note however that the notation in [10] is slightly different from ours. The constant $\tau$ used in [10] is equal to $\frac{2}{r_{1}^{2}+r_{2}^{2}} \tau$, the constant $D$ in [10] is equal to $\frac{\sqrt{r_{1}}}{\sqrt{r_{2}}} \gamma$ and $\sigma$ is used instead of $t$.

It is known that the Hastings-McLeod solution of Painlevé II has no poles on the real line [21]. Therefore the linear system (2.8) is well-defined for every choice of real parameters $r_{1}, r_{2}, s_{1}, s_{2}$, and $\tau$.

There are also linear differential equations

$$
\frac{\partial}{\partial s_{1}} M=V_{1} M, \quad \frac{\partial}{\partial s_{2}} M=V_{2} M, \quad \frac{\partial}{\partial \tau} M=W M,
$$

with explicitly known matrices $V_{1}, V_{2}$ and $W$. The Painlevé II equation (2.5) arises as the compatibility condition for (2.7) and (2.12), which can be viewed as a Lax pair. Note that the usual Lax pair for Painlevé II is of size $2 \times 2$, see $[18,19]$, and also section 2.6 below.

From (2.7) it follows that each column of $M$ is a solution to the linear system of ODEs

$$
\frac{\partial m}{\partial z}=U m, \quad m=\left(\begin{array}{llll}
m_{1} & m_{2} & m_{3} & m_{4}
\end{array}\right)^{T}
$$

with $U$ given by (2.8). To specify a solution of (2.13) it is enough to give $m_{1}$ and $m_{2}$ since

$$
\begin{aligned}
& i r_{1} m_{3}=m_{1}^{\prime}-\left(\tau-s_{1}^{2}+\frac{u}{C}\right) m_{1}-\frac{\sqrt{r_{2}} q}{\gamma \sqrt{r_{1}} C} m_{2}, \\
& i r_{2} m_{4}=m_{2}^{\prime}+\gamma \frac{\sqrt{r_{1}} q}{\sqrt{r_{2}} C} m_{1}-\left(-\tau+s_{2}^{2}-\frac{u}{C}\right) m_{2},
\end{aligned}
$$

which follows from the special structure of $U$ in (2.8).

\subsection{Tracy-Widom functions}

We are going to construct six solutions $m^{(j)}, j=0,1, \ldots, 5$, of (2.13). The formulas are based on functions that were first introduced by Tracy and Widom $[25,26]$.

For $t \in \mathbb{R}$, we use $K_{t}$ to denote the integral operator on $[t, \infty)$ with the Airy kernel

$$
\frac{\operatorname{Ai}(x) \operatorname{Ai}^{\prime}(y)-\operatorname{Ai}^{\prime}(x) \operatorname{Ai}(y)}{x-y} .
$$


Thus $K_{t}: L^{2}([t, \infty)) \rightarrow L^{2}([t, \infty))$ is defined by

$$
\left(K_{t} f\right)(x)=\int_{t}^{\infty} \frac{\operatorname{Ai}(x) \operatorname{Ai}^{\prime}(y)-\operatorname{Ai}^{\prime}(x) \operatorname{Ai}(y)}{x-y} f(y) d y .
$$

It is known that $I-K_{t}$ is invertible on $L^{2}([t, \infty))$, and we define two functions by

$$
\begin{aligned}
Q_{t} & =\left(I-K_{t}\right)^{-1} \mathrm{Ai}, \\
P_{t} & =\left(I-K_{t}\right)^{-1} \mathrm{Ai}^{\prime} .
\end{aligned}
$$

We also put

$$
R_{t}(x, y)=\frac{Q_{t}(x) P_{t}(y)-P_{t}(x) Q_{t}(y)}{x-y} .
$$

Both $Q_{t}$ and $P_{t}$ are continuous (in fact real analytic) functions on $[t, \infty)$. It is known that

$$
Q_{t}(t)=q(t), \quad R_{t}(t, t)=u(t),
$$

where $q$ is the Hastings-McLeod solution of (2.5) and $u$ is given by (2.6), see e.g. section 2.3 in [26] and in particular formula (25).

Lemma 2.4. Both $Q_{t}$ and $P_{t}$ extend to entire functions on the complex plane and

$$
\begin{aligned}
& Q_{t}(x)=\left(1+O\left(x^{-1 / 2}\right)\right) \operatorname{Ai}(x)=O\left(x^{-1 / 4} e^{-\frac{2}{3} x^{3 / 2}}\right), \\
& P_{t}(x)=\left(1+O\left(x^{-1}\right)\right) \operatorname{Ai}^{\prime}(x)=O\left(x^{1 / 4} e^{-\frac{2}{3} x^{3 / 2}}\right),
\end{aligned}
$$

as $x \rightarrow \infty$, uniformly for $-\pi+\varepsilon<\arg x<\pi-\varepsilon$, for every $\varepsilon>0$.

Proof. According to the Its-Izergin-Korepin-Slavnov theory [22] on integrable operators $\mathrm{C}$ (see also [8]) we have that

$$
\left(\begin{array}{c}
Q_{t}(x) \\
P_{t}(x)
\end{array}\right)=Y_{+}(x)\left(\begin{array}{c}
\operatorname{Ai}(x) \\
\operatorname{Ai}^{\prime}(x)
\end{array}\right)=Y_{-}(x)\left(\begin{array}{c}
\operatorname{Ai}(x) \\
\operatorname{Ai}^{\prime}(x)
\end{array}\right), \quad x \in(t, \infty) .
$$

where $Y$ is the unique solution of the $\mathrm{RH}$ problem:

- $Y: \mathbb{C} \backslash[t, \infty) \rightarrow \mathbb{C}^{2 \times 2}$ is analytic,

- $Y$ has continuous boundary values $Y_{+}$and $Y_{-}$on $(t, \infty)$ that satisfy

$$
Y_{+}(x)=Y_{-}(x)\left(I-2 \pi i\left(\begin{array}{c}
\operatorname{Ai}(x) \\
\operatorname{Ai}^{\prime}(x)
\end{array}\right)\left(\operatorname{Ai}^{\prime}(x)-\operatorname{Ai}(x)\right)\right),
$$

for $x \in(t, \infty)$, 
- $Y(x)=O(\log |x-t|)$ as $x \rightarrow t$,

- $Y(x)=I+O(1 / x)$ as $x \rightarrow \infty$.

Thus $x \in \mathbb{C} \mapsto Y(x)\left(\begin{array}{c}\operatorname{Ai}(x) \\ \operatorname{Ai}^{\prime}(x)\end{array}\right)$ provides the extension of $\left(\begin{array}{c}Q_{t} \\ P_{t}\end{array}\right)$ into an entire function on the complex plane. The lemma then follows because of the asymptotic behavior of $Y$ and the well known behavior

$$
\begin{aligned}
\operatorname{Ai}(x) & =\frac{1}{2 \sqrt{\pi} x^{1 / 4}} e^{-\frac{2}{3} x^{3 / 2}}\left(1+O\left(x^{-3 / 2}\right)\right) \\
\operatorname{Ai}^{\prime}(x) & =\frac{-x^{1 / 4}}{2 \sqrt{\pi}} e^{-\frac{2}{3} x^{3 / 2}}\left(1+O\left(x^{-3 / 2}\right)\right)
\end{aligned}
$$

as $x \rightarrow \infty,|\arg x|<\pi-\varepsilon$ of the Airy function.

By (2.20) we then also have that $R_{t}$ extends to an entire function in the complex plane and for every fixed $y$,

$$
R_{t}(x, y)=O\left(x^{-3 / 4} e^{-\frac{2}{3} x^{3 / 2}}\right)
$$

as $x \rightarrow \infty$ with $-\pi+\varepsilon<\arg x<\pi-\varepsilon$ for some $\varepsilon>0$.

\subsection{Six solutions of (2.13)}

The functions $Q_{t}(x)$ and $R_{t}(x, t)$ from (2.18) and (2.20) appear explicitly in the integral formulas we have for the solutions of (2.13). We use

$$
\omega=e^{2 \pi i / 3} .
$$

We also put

$$
\lambda=\frac{r_{2}^{2}-r_{1}^{2}}{r_{1}^{2}+r_{2}^{2}} \tau, \quad \mu=\frac{2}{r_{1}^{2}+r_{2}^{2}} \tau
$$

and we recall the definitions of $C, \gamma$ and $t$ in (2.9), (2.10) and (2.11).

Theorem 2.5. There are six solutions $m^{(j)}, j=0, \ldots, 5$ of (2.13) whose first and second components are given below. In all cases the third and fourth components are as in (2.14)-(2.15).

The six solutions are given as follows. 
Solution $m^{(0)}$ Let $F_{0}(z)=\operatorname{Ai}\left(r_{2}^{2 / 3} z+\frac{2 s_{2}}{r_{2}^{1 / 3}}\right) e^{-r_{2}^{2} \mu z}$. Then

$$
\left(\begin{array}{l}
m_{1}^{(0)}(z) \\
m_{2}^{(0)}(z)
\end{array}\right)=\sqrt{2 \pi} r_{2}^{1 / 6} e^{\lambda z}\left(\begin{array}{c}
-\frac{\sqrt{r_{2}}}{\gamma \sqrt{r_{1}}} \int_{t}^{\infty} F_{0}(z+C(x-t)) Q_{t}(x) d x \\
F_{0}(z)+\int_{t}^{\infty} F_{0}(z+C(x-t)) R_{t}(x, t) d x
\end{array}\right) .
$$

Solution $m^{(1)}$ Let $F_{1}(z)=\omega \operatorname{Ai}\left(\omega\left(r_{1}^{2 / 3} z+\frac{2 s_{1}}{r_{1}^{1 / 3}}\right)\right) e^{-r_{1}^{2} \mu z}$. Then

$$
\left(\begin{array}{l}
m_{1}^{(1)}(z) \\
m_{2}^{(1)}(z)
\end{array}\right)=-\sqrt{2 \pi} r_{1}^{1 / 6} e^{\lambda z}\left(\begin{array}{c}
F_{1}(-z)+\int_{t}^{\infty \omega^{2}} F_{1}(-z+C(x-t)) R_{t}(x, t) d x \\
-\gamma \frac{\sqrt{r_{1}}}{\sqrt{r_{2}}} \int_{t}^{\infty \omega^{2}} F_{1}(-z+C(x-t)) Q_{t}(x) d x
\end{array}\right) .
$$

Solution $m^{(2)}$ Let $F_{2}(z)=\omega^{2} \operatorname{Ai}\left(\omega^{2}\left(r_{2}^{2 / 3} z+\frac{2 s_{2}}{r_{2}^{1 / 3}}\right)\right) e^{-r_{2}^{2} \mu z}$. Then

$$
\left(\begin{array}{l}
m_{1}^{(2)}(z) \\
m_{2}^{(2)}(z)
\end{array}\right)=-\sqrt{2 \pi} r_{2}^{1 / 6} e^{\lambda z}\left(\begin{array}{c}
-\frac{\sqrt{r_{2}}}{\gamma \sqrt{r_{1}}} \int_{t}^{\infty \omega} F_{2}(z+C(x-t)) Q_{t}(x) d x \\
F_{2}(z)+\int_{t}^{\infty \omega} F_{2}(z+C(x-t)) R_{t}(x, t) d x
\end{array}\right) .
$$

Solution $m^{(3)}$ Let $F_{3}(z)=\operatorname{Ai}\left(r_{1}^{2 / 3} z+\frac{2 s_{1}}{r_{1}^{1 / 3}}\right) e^{-r_{1}^{2} \mu z}$. Then

$$
\left(\begin{array}{l}
m_{1}^{(3)}(z) \\
m_{2}^{(3)}(z)
\end{array}\right)=\sqrt{2 \pi} r_{1}^{1 / 6} e^{\lambda z}\left(\begin{array}{c}
F_{3}(-z)+\int_{t}^{\infty} F_{3}(-z+C(x-t)) R_{t}(x, t) d x \\
-\gamma \frac{\sqrt{r_{1}}}{\sqrt{r_{2}}} \int_{t}^{\infty} F_{3}(-z+C(x-t)) Q_{t}(x) d x
\end{array}\right) .
$$

Solution $m^{(4)}$ Let $F_{4}(z)=\omega \operatorname{Ai}\left(\omega\left(r_{2}^{2 / 3} z+\frac{2 s_{2}}{r_{2}^{1 / 3}}\right)\right) e^{-r_{2}^{2} \mu z}$. Then

$$
\left(\begin{array}{l}
m_{1}^{(4)}(z) \\
m_{2}^{(4)}(z)
\end{array}\right)=\sqrt{2 \pi} r_{2}^{1 / 6} e^{\lambda z}\left(\begin{array}{c}
-\frac{\sqrt{r_{2}}}{\gamma \sqrt{r_{1}}} \int_{t}^{\infty \omega^{2}} F_{4}(z+C(x-t)) Q_{t}(x) d x \\
F_{4}(z)+\int_{t}^{\infty \omega^{2}} F_{4}(z+C(x-t)) R_{t}(x, t) d x
\end{array}\right) .
$$

Solution $m^{(5)}$ Let $F_{5}(z)=\omega^{2} \operatorname{Ai}\left(\omega^{2}\left(r_{1}^{2 / 3} z+\frac{2 s_{1}}{r_{1}^{1 / 3}}\right)\right) e^{-r_{1}^{2} \mu z}$. Then

$$
\left(\begin{array}{c}
m_{1}^{(5)}(z) \\
m_{2}^{(5)}(z)
\end{array}\right)=\sqrt{2 \pi} r_{1}^{1 / 6} e^{\lambda z}\left(\begin{array}{c}
F_{5}(-z)+\int_{t}^{\infty \omega} F_{5}(-z+C(x-t)) R_{t}(x, t) d x \\
-\gamma \frac{\sqrt{r_{1}}}{\sqrt{r_{2}}} \int_{t}^{\infty \omega} F_{5}(-z+C(x-t)) Q_{t}(x) d x
\end{array}\right) .
$$


The proof of Theorem 2.5 is given in section 3.3 .

Remark 2.6. The solutions $m^{(0)}$ and $m^{(3)}$ were found by Delvaux [10]. These are the solutions for which the integrals are taken over the real interval $(t, \infty)$. The other solutions are new and their identification is the main result of this paper.

Remark 2.7. The integrals in (2.31) and (2.34) start at $t \in \mathbb{R}$ and end at infinity at asymptotic angle $2 \pi / 3$. Similary, the integrals in (2.30) and (2.33) end at asymptotic angle $-2 \pi / 3$.

By Lemma 2.4 we have that $Q_{t}(x)$ and $R_{t}(x, t)$ are entire functions in $x$. To see that the integrals in (2.31) indeed converge, we note that both $Q_{t}(x)=O\left(e^{-\frac{2}{3} x^{3 / 2}}\right)$ and $R_{t}(x, t)=O\left(e^{-\frac{2}{3} x^{3 / 2}}\right)$ by $(2.22)$ and $(2.27)$, and so

$$
F_{2}(z+C(x-t))=e^{\frac{2}{3}\left(r_{2} C^{3 / 2} x^{3 / 2}\right)+O(x)}
$$

as $x \rightarrow \infty$ with $\arg x=2 \pi / 3$. Since by $(2.9)$

$$
r_{2} C^{3 / 2}=r_{2}\left(r_{1}^{-2}+r_{2}^{-2}\right)^{1 / 2}>1
$$

the integrands decay at an exponential rate at infinity, and the integrals in (2.31) converge.

Similarly, the integrals in (2.30), (2.33) and (2.34) converge.

\subsection{The solution of the tacnode $\mathrm{RH}$ problem}

The vector $m^{(j)}, j=0, \ldots, 5$ turns out to be the recessive solution of $(2.13)$ in the sector

$$
S_{j}=\left\{z \in \mathbb{C} \mid-\frac{\pi}{6}+j \frac{\pi}{3}<\arg z<\frac{\pi}{6}+j \frac{\pi}{3}\right\} .
$$

Note that $S_{j}$ is the sector of angular width $\pi / 3$ with $\Gamma_{j}$ as its bisector, see (2.1). The constant prefactors in the definitions (2.29)-(2.34) are chosen such that $m^{(j)}$ appears as one of the columns of $M$ in the two sectors $\Omega_{j-1}$ and $\Omega_{j}$ that intersect $S_{j}$, where it is understood that $\Omega_{-1}=\Omega_{5}$. As such the vectors $m^{(j)}$ are the building blocks for the solution of the tacnode $\mathrm{RH}$ problem.

Our main result is the following.

Theorem 2.8. The solution of the tacnode $R H$ problem (2.2)-(2.4) is given by

$$
M=\left(\begin{array}{llll}
m^{(3)} & m^{(0)} & m^{(1)} & m^{(2)}
\end{array}\right)
$$




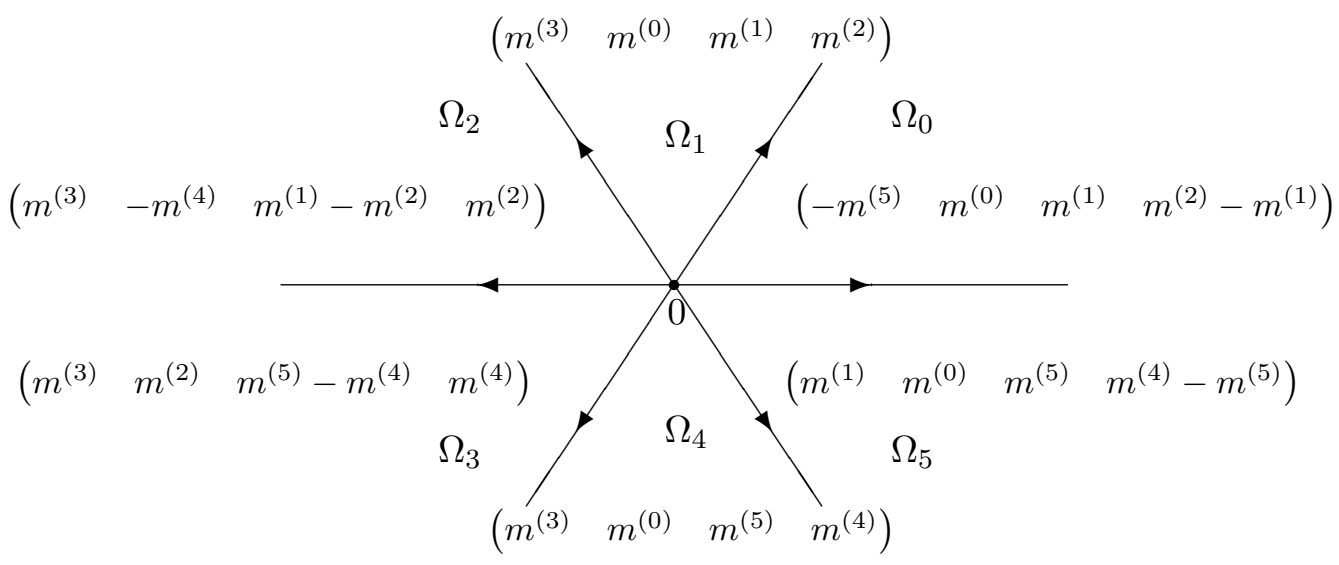

Figure 2: Structure of the solution of the tacnode RH problem. The column vector $m^{(j)}$ is the recessive solution of $(2.13)$ in the sector $S_{j}$.

in the sector $\pi / 3<\arg z<2 \pi / 3$ around the positive imaginary axis. In the other sectors it can be found by following the jumps (2.2) in the RH problem, and by using the non-trivial relations

$$
m^{(0)}+m^{(3)}=m^{(1)}-m^{(5)}=m^{(2)}-m^{(4)}
$$

among the six solutions $m^{(0)}, \ldots, m^{(5)}$ of $(2.13)$.

Explicit expressions for $M$ in all sectors $\Omega_{j}$ are given in Figure 2.

The proof of Theorem 2.8 is given in section 3.4.

The relations (2.36) among the recessive solutions of (2.13) are quite remarkable as they do not follow in a straightforward way from the integral representations. It is an open problem how to prove these identities in a direct way from the formulas (2.29)-(2.34).

\subsection{Comparison with the $2 \times 2 \mathrm{RH}$ problem for Painlevé II}

The usual RH problem for Painlevé II is of size $2 \times 2$ with a contour $\Gamma_{\Psi}$ as in Figure 3. Then $\Psi: \mathbb{C} \backslash \Gamma_{\Psi} \rightarrow \mathbb{C}^{2 \times 2}$ is analytic satisfying $\Psi_{+}=\Psi_{-} J_{\Psi}$ on $\Gamma_{\Psi}$ with jump matrices $J_{\Psi}$ that are also shown in Figure 3 , and with the 


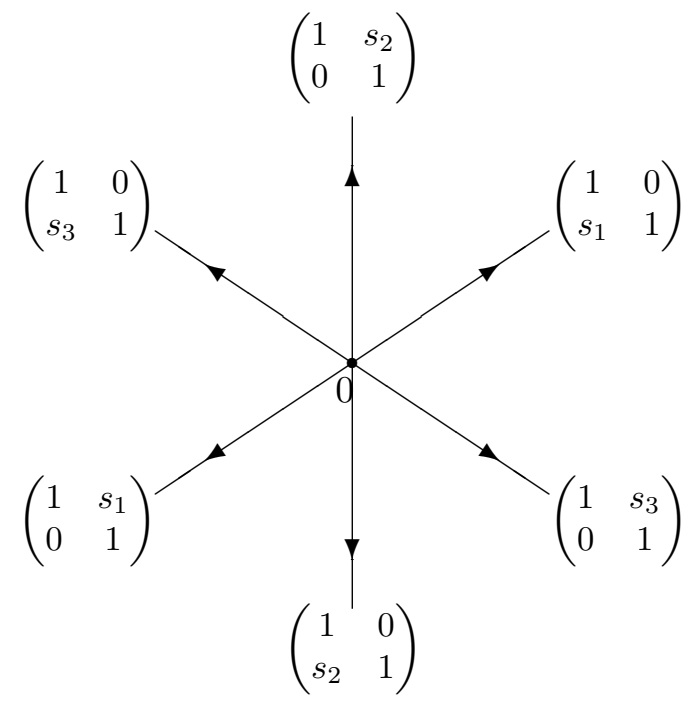

Figure 3: Jump matrices in the $2 \times 2 \mathrm{RH}$ problem for Painlevé II. The Stokes multipliers satisfy $s_{1} s_{2} s_{3}+s_{1}+s_{2}+s_{3}=0$. The Hastings McLeod solution corresponds to $s_{1}=1, s_{2}=0$ and $s_{3}=-1$.

asymptotic condition

$$
\Psi(z)=(I+O(1 / z)) e^{-i\left(\frac{4}{3} z^{3}+t z\right) \sigma_{3}}, \quad \text { as } z \rightarrow \infty
$$

where $\sigma_{3}=\left(\begin{array}{cc}1 & 0 \\ 0 & -1\end{array}\right)$. The Stokes multipliers $s_{1}, s_{2}, s_{3}$ that appear in the jump matrices are assumed to satisfy $s_{1} s_{2} s_{3}+s_{1}+s_{2}+s_{3}=0$. Then a unique solution to the $\mathrm{RH}$ problem exists (except for an at most countable number of $t$ values), and $\Psi$ satisfies the differential equations (Lax pair)

$$
\begin{aligned}
\frac{\partial}{\partial z} \Psi & =\left(\begin{array}{cc}
-4 i z^{2}-i\left(t+2 q^{2}\right) & 4 z q+2 i q^{\prime} \\
4 z q-2 i q^{\prime} & 4 i z^{2}+i\left(t+2 q^{2}\right)
\end{array}\right) \Psi \\
\frac{\partial}{\partial t} \Psi & =\left(\begin{array}{cc}
-i z & q \\
q & i z
\end{array}\right) \Psi
\end{aligned}
$$

where $q=q(t)$ is a solution of the Painlevé II equation, determined by the Stokes multipliers, see e.g. [18, 19]. 


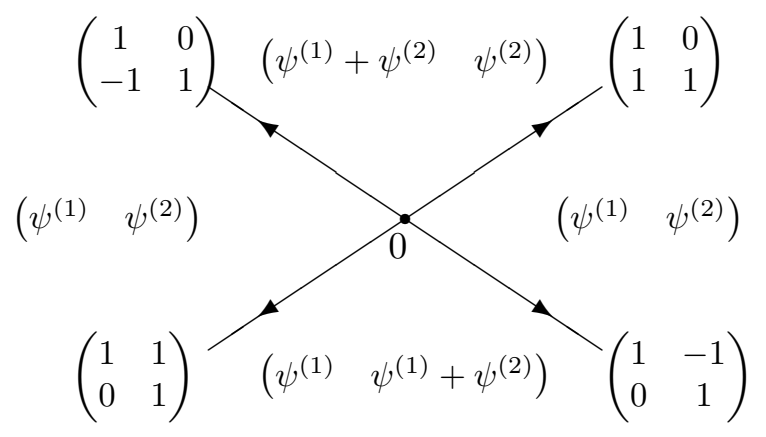

Figure 4: Solution of the $2 \times 2 \mathrm{RH}$ problem for the Hastings-McLeod solution of Painlevé II in terms of the columnn vectors $\psi^{(1)}$ and $\psi^{(2)}$.

The special case $s_{1}=1, s_{2}=0, s_{3}=-1$ leads to the Hastings McLeod solution of the Painlevé II equation. In this case there is an explicit formula for the solution of the $\mathrm{RH}$ problem, which is contained in the recent work of Baik, Liechty, and Schehr [6], see also [5]. The solution is built out of the two column vectors

$$
\begin{aligned}
& \psi^{(1)}(z)=e^{-i\left(\frac{4}{3} z^{3}+t z\right)}\left(\begin{array}{c}
1+\int_{t}^{\infty} e^{-2 i(x-t) z} R_{t}(x, t) d x \\
-\int_{t}^{\infty} e^{-2 i(x-t) z} Q_{t}(x) d x
\end{array}\right), \\
& \psi^{(2)}(z)=e^{i\left(\frac{4}{3} z^{3}+t z\right)}\left(\begin{array}{c}
-\int_{t}^{\infty} e^{2 i(x-t) z} Q_{t}(x) d x \\
1+\int_{t}^{\infty} e^{2 i(x-t) z} R_{t}(x, t) d x
\end{array}\right),
\end{aligned}
$$

that satisfy the vector analogue of (2.37). Then the solution of the $\mathrm{RH}$ problem is

$$
\Psi(z)=\left(\begin{array}{ll}
\psi^{(1)} & \psi^{(2)}
\end{array}\right)
$$

in the two sectors $-\frac{\pi}{6}<\arg z<\frac{\pi}{6}$ and $\frac{5 \pi}{6}<\arg z<\frac{7 \pi}{6}$. The solution in all sectors is given in Figure 4. 
Comparing this solution with the explicit solution $M$ of the $4 \times 4$ matrixvalued $\mathrm{RH}$ problem, we see that the same functions $Q_{t}(x)$ and $R_{t}(x, t)$ appear in the solutions, but there does not seem to be a direct way to go from $\Psi$ to $M$. The $4 \times 4 \mathrm{RH}$ problem and its associated Lax pair therefore provide a genuinely different characterization of the Hastings-McLeod solution of Painlevé II.

\subsection{Critical kernel in random matrix theory}

As already mentioned, there are two correlation kernels in random matrix theory that can be expressed in terms of the tacnode $\mathrm{RH}$ problem, namely the one-time correlation kernel for the tacnode process [12], and a critical kernel in the two-matrix model [13]. The implications of the explicit form of the solution of the tacnode $\mathrm{RH}$ problem for the tacnode process was discussed by Delvaux [10], who made the connection between [12] and the different sets of formulas derived in $[2,3,17,23]$. We briefly discuss it in the section 4.1.

The critical kernel in the two-matrix model is due to Duits and Geudens [13]. Here the setting is as follows. The two-matrix model is the probability measure

$$
\frac{1}{Z_{n}} \exp \left(-n \operatorname{Tr}\left(V\left(M_{1}\right)+W\left(M_{2}\right)-\tau M_{1} M_{2}\right)\right) d M_{1} d M_{2}
$$

defined on pairs $\left(M_{1}, M_{2}\right)$ of $n \times n$ Hermitian matrices, where $\tau$ is the coupling constant. The model is determinantal in the sense that the combined eigenvalues of $M_{1}$ and $M_{2}$ are a determinantal point process, see [16].

In [13] the case

$$
V(x)=\frac{1}{2} x^{2}, \quad W(y)=\frac{1}{2} y^{4}+\frac{\alpha}{2} y^{2}
$$

is considered. For these potentials the correlation kernel of the eigenvalues of $M_{1}$, when averaged over $M_{2}$, is expressible in terms of a Riemann-Hilbert problem of size $4 \times 4$ that depends on the two parameters $\alpha$ and $\tau$ in the model. A complete study is made of the critical phenomena and phase transitions that take place in the $\alpha-\tau$ phase diagram as $n \rightarrow \infty$. It turns out that there is a multi-critical point corresponding to the values

$$
\alpha=-1 \quad \text { and } \quad \tau=1,
$$

see also $[14,15]$. In a double scaling limit around these values the correlation kernel for the eigenvalues of $M_{1}$ leads to a two parameter family of 
new critical kernels that quite remarkably can be expressed in terms of the solution of the tacnode RH problem (2.2)-(2.4) with the special choice of parameters

$$
r_{1}=r_{2}=1, \quad s_{1}=s_{2}=s, \quad \tau \in \mathbb{R} .
$$

With those parameters we have by (2.9)-(2.11), and (2.28),

$$
C=2^{1 / 3}, \quad \gamma=1, \quad t=2^{2 / 3}\left(2 s-\tau^{2}\right), \quad \lambda=0, \quad \mu=\tau .
$$

The formula for the critical kernel with parameters $s$ and $\tau$ is

$$
K^{c r i t}(x, y ; s, \tau)=\frac{1}{2 \pi i(x-y)}\left(\begin{array}{llll}
-1 & 1 & 0 & 0
\end{array}\right) M(i x)^{-1} M(i y)\left(\begin{array}{l}
1 \\
1 \\
0 \\
0
\end{array}\right) \text {, }
$$

which can easily be obtained from the formulas (2.13) and (2.15) in [13]. Here $M$ is the solution of the tacnode $\mathrm{RH}$ problem with parameters (2.41). The final result of this paper is an explicit integral representation for this kernel.

Theorem 2.9. The Duits-Geudens critical kernel (2.43) is given by

$$
K^{c r i t}(x, y ; s, \tau)=\frac{2}{\pi} \int_{s}^{\infty} \operatorname{Im}\left[\widetilde{m}_{1}\left(i x, s^{\prime},-\tau\right) \widehat{m}_{1}\left(i y, s^{\prime}, \tau\right)\right] d s^{\prime}
$$

with

$$
\widetilde{m}_{1}=m_{1}^{(1)}-m_{1}^{(2)}, \quad \widehat{m}_{1}=m_{1}^{(0)}+m_{1}^{(3)},
$$

where $m_{1}^{(0)}, \ldots, m_{1}^{(3)}$ are the first components of the vectors given in (2.29)(2.32) with the special values (2.41)-(2.42) for the parameters.

Theorem 2.9 is proved in section 4.2 .

\section{Proofs of Theorems 2.5 and 2.8}

\subsection{Transformation to second order system}

It will be convenient to transform the first order system (2.13) to a second order system. The transformation also removes the parameter $\gamma$.

Lemma 3.1. Suppose $m=\left(\begin{array}{llll}m_{1} & m_{2} & m_{3} & m_{4}\end{array}\right)^{T}$ satisfies (2.13). Then

$$
\psi(z)=\left(\begin{array}{c}
\psi_{1}(z) \\
\psi_{2}(z)
\end{array}\right)=e^{-\lambda z}\left(\begin{array}{c}
\gamma \frac{\sqrt{r_{1}}}{\sqrt{r_{2}}} m_{1}(z) \\
m_{2}(z)
\end{array}\right)
$$


satisfies

$$
\frac{\partial^{2} \psi}{\partial z^{2}}=A \frac{\partial \psi}{\partial z}+B \psi
$$

where

$$
\begin{aligned}
A & =\left(\begin{array}{cc}
2 r_{1}^{2} \mu & C^{2} r_{1}^{2} q \\
-C^{2} r_{2}^{2} q & -2 r_{2}^{2} \mu
\end{array}\right) \\
B & =\left(\begin{array}{cc}
-r_{1}^{2} z+2 r_{1} s_{1}+C r_{1}^{2} q^{2}-r_{1}^{4} \mu^{2} & -C r_{1}^{2} q^{\prime} \\
-C r_{2}^{2} q^{\prime} & r_{2}^{2} z+2 r_{2} s_{2}+C r_{2}^{2} q^{2}-r_{2}^{4} \mu^{2}
\end{array}\right) .
\end{aligned}
$$

Conversely, if $\psi=\left(\psi_{1}, \psi_{2}\right)^{T}$ satisfies (3.2) with $A$ and $B$ given by (3.3)(3.4) then $m=\left(m_{1}, m_{2}, m_{3}, m_{4}\right)^{T}$ where

$$
m_{1}(z)=e^{\lambda z} \gamma^{-1} \frac{\sqrt{r_{2}}}{\sqrt{r_{1}}} \psi_{1}(z), \quad m_{2}(z)=e^{\lambda z} \psi_{2}(z)
$$

and $m_{3}, m_{4}$ are given by (2.14)-(2.15) satisfies (2.13).

Proof. This is a straightforward calculation and we will not give full details, see also [10, Proposition 2.12].

Let us just note that for general $\lambda$ one obtains (3.2) with

$$
A=\left(\begin{array}{cc}
2 \tau-2 \lambda & C^{2} r_{1}^{2} q \\
-C^{2} r_{2}^{2} q & -2 \tau-2 \lambda
\end{array}\right)
$$

and

$B=\left(\begin{array}{cc}-r_{1}^{2} z+2 r_{1} s_{1}+C r_{1}^{2} q^{2}-(\tau-\lambda)^{2} & \left.-C r_{1}^{2} q^{\prime}+\left(\left(r_{1}^{2}-r_{2}^{2}\right) \tau+\left(r_{1}^{2}+r_{2}^{2}\right) \lambda\right)\right) \frac{q}{r_{2}^{2} C} \\ \left.-C r_{2}^{2} q^{\prime}-\left(\left(r_{1}^{2}-r_{2}^{2}\right) \tau+\left(r_{1}^{2}+r_{2}^{2}\right) \lambda\right)\right) \frac{q}{r_{1}^{2} C} & r_{2}^{2} z+2 r_{2} s_{2}+C r_{2}^{2} q^{2}-(\tau+\lambda)^{2}\end{array}\right)$

Because of the choice (2.28) for $\lambda$ we have that the off-diagonal entries of $B$ simplify since the terms with $q$ disappear. By (2.28) it is easy to check that

$$
\tau-\lambda=r_{1}^{2} \mu, \quad \tau+\lambda=r_{2}^{2} \mu
$$

and we obtain (3.3) and (3.4).

\subsection{Solutions to the second order system (3.2)}

We denote $\omega=e^{2 \pi i / 3}$ as before, and we let

$$
y_{0}(x)=\operatorname{Ai}(x), \quad y_{1}(x)=\omega \operatorname{Ai}(\omega x), \quad y_{2}(x)=\omega^{2} \operatorname{Ai}\left(\omega^{2} x\right)
$$

be three solutions of the Airy differential equation $y^{\prime \prime}=x y$.

The following lemma gives solutions to the second order system (3.2). 
Lemma 3.2. For $k=0,1,2$, we put

$$
F(z)=y_{k}\left(r_{2}^{2 / 3} z+\frac{2 s_{2}}{r_{2}^{1 / 3}}\right) \exp \left(-r_{2}^{2} \mu z\right) .
$$

Then the vector $\psi=\left(\begin{array}{l}\psi_{1} \\ \psi_{2}\end{array}\right)$ where

$$
\begin{aligned}
& \psi_{1}(z)=-\int_{t}^{\infty \omega^{2 k}} F(z+C(x-t)) Q_{t}(x) d x, \\
& \psi_{2}(z)=F(z)+\int_{t}^{\infty \omega^{2 k}} F(z+C(x-t)) R_{t}(x, t) d x,
\end{aligned}
$$

is a solution of (3.2).

Proof. For $k=0$, this is proved in [10, section 5.2], although with somewhat different notation. The proof uses the differential equation for $F$

$$
F^{\prime \prime}(z)=-2 r_{2}^{2} \mu F^{\prime}(z)+\left(r_{2}^{2} z+2 r_{2} s_{2}-r_{2}^{4} \mu^{2}\right) F(z),
$$

see also [10, Equ. (5.2)] (where $F$ is called $b_{z}(x)$ and $\mu$ is called $\tau$ ), and the fact that the integrals in (3.7) and (3.8) converge for $k=0$. We already noted, see Remark 1.6, that the integrals in (3.7) and (3.8) converge for $k=1,2$ as well.

What is also used in [10] are a number of identities for the functions $Q_{t}$, $P_{t}$ and $R_{t}$ introduced in (2.18)-(2.20), namely the differential identities (see also $[25,26]$ or $[4$, section 3.8$])$

$$
\begin{aligned}
\frac{\partial}{\partial t} R_{t}(x, y) & =-R_{t}(x, t) R_{t}(t, y), \\
\frac{\partial}{\partial t} Q_{t}(x) & =-R_{t}(x, t) Q_{t}(x), \\
\frac{\partial}{\partial t} P_{t}(x) & =-R_{t}(x, t) P_{t}(x),
\end{aligned}
$$

and identities for the $x$-derivatives of $Q_{t}$ and $P_{t}$,

$$
\begin{aligned}
& Q_{t}^{\prime}(x)=P_{t}(x)+q(t) R_{t}(x, t)-u(t) Q_{t}(x), \\
& P_{t}^{\prime}(x)=x Q_{t}(x)+p(t) R_{t}(x, t)+u(t) P_{t}(x)-2 v(t) Q_{t}(x) .
\end{aligned}
$$

The identities (3.10)-(3.14) of course extend into the complex plane. 
Also for $k=1,2$, we have that $F$ satisfies (3.9). Since the integrals in (3.7) and (3.8) converge for $k=1,2$, and the identities (3.10)-(3.12) and (3.13)-(3.14) remain valid for $x$ in the complex plane, we can follow the proof in [10, section 5.2], making proper modifications due to some change in notation. For convenience of the reader we provide the detailed calculations in the appendix (section 5) using the notations of this paper.

\subsection{Proof of Theorem 2.5}

We can now prove Theorem 2.5

Proof. In view of Lemmas 3.1 and 3.2 we find three solutions of (2.13). After multiplication by appropriate constants these are the solutions $m^{(0)}, m^{(2)}$ and $m^{(4)}$ given by the formulas (2.29), (2.31), (2.33) in Theorem 2.5.

The other solutions follow from a symmetry in the system (2.13). Namely, if $m(z)$ is a solution of (2.13) then

$$
\left(\begin{array}{cc}
J & O \\
O & -J
\end{array}\right) m(-z), \quad J=\left(\begin{array}{cc}
0 & 1 \\
1 & 0
\end{array}\right)
$$

solves (2.13) as well, but with the change of parameters

$$
r_{1} \leftrightarrow r_{2}, \quad s_{1} \leftrightarrow s_{2}, \quad \tau \mapsto \tau .
$$

The constants $C, t$ and $\mu$ do not change under this change of parameters, but

$$
\gamma \mapsto \gamma^{-1}, \quad \lambda \mapsto-\lambda,
$$

see the formulas (2.9)-(2.11) and (2.28). Thus one solution of (2.13) leads to another by a change of sign $z \mapsto-z$, a change of parameters (3.15), combined with an interchange $m_{1} \leftrightarrow m_{2}$. In this way the solutions $m^{(0)}$, $m^{(2)}$ and $m^{(4)}$ lead to the solutions $m^{(3)}, m^{(5)}$, and $m^{(1)}$.

This completes the proof of Theorem 2.5.

\subsection{Proof of Theorem 2.8}

Proof. The column vectors of $M$ are in each sector $\Omega_{k}$ a basis of the vector space of solutions of (2.13). In each sector the four different columns represent the four different types of asymptotic behavior, as given by (2.4). 
Namely, if $e_{k}$ denotes the $k$ th unit vector,

$$
\begin{aligned}
& M(z) e_{1} \sim e^{-\theta_{1}(z)+\tau z} \\
& M(z) e_{2} \sim e^{-\theta_{2}(z)-\tau z} \\
& M(z) e_{3} \sim e^{\theta_{1}(z)+\tau z} \\
& M(z) e_{4} \sim e^{\theta_{2}(z)-\tau z}
\end{aligned}
$$

as $z \rightarrow \infty$.

Distinguished solutions of (2.13) are those solutions that are recessive in a certain sector, i.e., they are smallest possible as $z \rightarrow \infty$ in that sector. Recessive solutions are unique up to a multiplicative constant. From (3.16) and (2.3) one sees that $M(z) e_{1}$ is a recessive solution of (2.13) in the sector $S_{3}, M(z) e_{2}$ is a recessive solution in $S_{0}, M(z) e_{3}$ is a recessive solution in sectors $S_{1}$ and $S_{5}$ and $M(z) e_{4}$ is recessive in sectors $S_{2}$ and $S_{4}$.

It will turn out that $m^{(j)}$ is the recessive solution of (2.13) in sector $S_{j}$. This then implies, for example, that $m^{(0)}$ is a multiple of $M(z) e_{2}$ in $S_{0}$. The constant $\sqrt{2 \pi} r_{2}^{1 / 6}$ in the definition (2.29) of $m^{(0)}$ has been chosen so that $M(z) e_{2}=m^{(0)}$ in $S_{0}$. Since $S_{0}$ has a non-empty intersection with both $\Omega_{0}$ and $\Omega_{5}$ it then follows that $m^{(0)}$ appears as the second column of $M$ in sectors $\Omega_{0}$ and $\Omega_{5}$.

Similarly, $m^{(1)}$ is in the third column of $M$ in the sectors $\Omega_{0}$ and $\Omega_{1}$, $m^{(2)}$ is in the fourth column of $M$ in $\Omega_{1}$ and $\Omega_{2}$, and so on.

This leads to the partial solution of the $\mathrm{RH}$ problem given in Figure 5.

Then we complete the solution by following the effect of the jump matrices $J_{k}$, see Figure 1, and this leads to the full solution of the tacnode $\mathrm{RH}$ problem as given in Figure 2.

We prove in more detail that $m^{(0)}$ is the recessive solution of (2.13) in the sector $S^{(0)}$ and that $m^{(0)}=M e_{2}$ in $\Omega_{0} \cup \Omega_{5}$. The arguments for the other solutions $m^{(j)}$ can be done in a similar way.

The asymptotic condition (2.4) tells us that

$$
M_{22}(z)=\left(\frac{1}{\sqrt{2}} z^{-1 / 4}+O\left(z^{-3 / 4}\right)\right) e^{-\theta_{2}(z)-\tau z}
$$

as $z \rightarrow \infty$. It will be enough to show that $m_{2}^{(0)}$ has the same asymptotic behavior as $z \rightarrow \infty$ in the sector $S_{0}$ in order to conclude that $m^{(0)}=M e_{2}$ in $\Omega_{0} \cup \Omega_{5}$. 


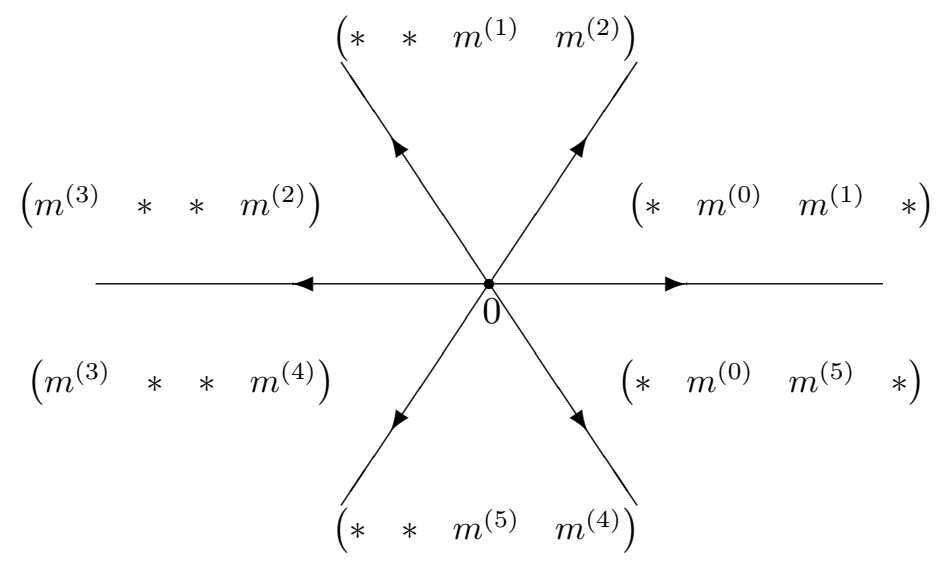

Figure 5: Partial solution of the tacnode $\mathrm{RH}$ problem with only those columns that are recessive solutions of (2.13) in parts of certain sectors. The $*$ entries denote columns that still have to be determined.

Recall that $m_{2}^{(0)}$ is given in (2.29). Since $\lambda-r_{2}^{2} \mu=-\tau$ we can write

$$
\begin{aligned}
& m_{2}^{(0)}(z)=\sqrt{2 \pi} r_{2}^{1 / 6} e^{-\tau z} \operatorname{Ai}\left(r_{2}^{2 / 3} z+\frac{2 s_{2}}{r_{2}^{1 / 3}}\right) \\
& +\sqrt{2 \pi} r_{2}^{1 / 6} e^{-\tau z} \int_{t}^{\infty} \operatorname{Ai}\left(r_{2}^{2 / 3}(z+C(x-t))+\frac{2 s_{2}}{r_{2}^{1 / 3}}\right) e^{-r_{2}^{2} C \mu(x-t)} R_{t}(x, t) d x .
\end{aligned}
$$

From the asymptotic behavior of the Airy function, see (2.26), we have as $z \rightarrow \infty$ with $|\arg z|<\pi-\varepsilon$,

$$
\operatorname{Ai}\left(r_{2}^{2 / 3} z+\frac{2 s_{2}}{r_{2}^{1 / 3}}\right)=\frac{1}{2 \sqrt{\pi} r_{2}^{1 / 6} z^{1 / 4}} e^{-\theta_{2}(z)}\left(1+O\left(z^{-1 / 2}\right)\right)
$$

(see $(2.3)$ for $\left.\theta_{2}\right)$, and

$\operatorname{Ai}\left(r_{2}^{2 / 3}(z+C(x-t))+\frac{2 s_{2}}{r_{2}^{1 / 3}}\right)=\frac{1}{2 \sqrt{\pi} r_{2}^{1 / 6} z^{1 / 4}} e^{-\theta_{2}(z)-r_{2} C(x-t) z^{1 / 2}}\left(1+O\left(z^{-1 / 2}\right)\right)$

with a $O\left(z^{-1 / 2}\right)$ term that is uniform for $x \geq t$ in case $|\arg z|<\pi / 6$. Thus 
the first term in the right-hand side of (3.18) is

$$
\left(\frac{1}{\sqrt{2}} z^{-1 / 4}+O\left(z^{-3 / 4}\right)\right) e^{-\theta_{2}(z)-\tau z}
$$

as $z \rightarrow \infty$, while the second term is (for $z \rightarrow \infty$ in $S_{0}$ )

$$
O\left(z^{-1 / 4}\right) e^{-\theta_{2}(z)-\tau z} \int_{t}^{\infty} e^{-r_{2} C(x-t) z^{1 / 2}} e^{-r_{2}^{2} C \mu(x-t)} R_{t}(x, t) d x .
$$

As $z \rightarrow \infty$ in $S_{0}$, the main contribution to the integral in (3.21) comes from the endpoint $x=t$. A crude form of Laplace's method, see e.g. [24], shows that the integral is $O\left(z^{-1 / 2}\right)$ and therefore we find that the second term in the right-hand side of $(3.18)$ is $O\left(z^{-3 / 4}\right) e^{-\theta_{2}(z)-\tau z}$. Together with (3.20) this gives

$$
m_{2}^{(0)}(z)=\left(\frac{1}{\sqrt{2}} z^{-1 / 4}+O\left(z^{-3 / 4}\right)\right) e^{-\theta_{2}(z)-\tau z}
$$

as $z \rightarrow \infty$ in $S_{0}$, which indeed agrees with (3.17).

This completes the proof of Theorem 2.8.

Remark 3.3. A more refined application of Laplace's method would lead to

$$
m_{2}^{(0)}(z)=\left(z^{-1 / 4}+\frac{u(t)}{r_{2} C} z^{-3 / 4}+\cdots\right) \frac{e^{-\theta_{2}(z)-\tau z}}{\sqrt{2}}
$$

and similarly

$m_{1}^{(0)}(z)=\left(-\frac{q(t)}{\gamma \sqrt{r_{1} r_{2}} C} z^{-3 / 4}+\frac{\left(r_{2}^{2} \mu+s_{2}^{2}\right) C q(t)-p(t)}{\gamma \sqrt{r_{1} r_{2}} r_{2} C^{2}} z^{-5 / 4}+O\left(z^{-7 / 4}\right)\right) \frac{e^{-\theta_{2}(z)-\tau z}}{\sqrt{2}}$

where it is used that $Q_{t}(t)=q(t), Q_{t}^{\prime}(t)=p(t)$ and $R_{t}(t, t)=u(t)$. This leads to formulas for certain entries in $M^{(1)}$ (this is the residue matrix from the asymptotic condition, see (2.4)), but we do not discuss that here.

\section{Correlation kernels}

As already mentioned in the introduction, there are two correlation kernels in random matrix theory that can be expressed in terms of the tacnode $\mathrm{RH}$ problem, namely the one-time correlation kernel for the tacnode process [12], and a critical kernel in the two-matrix model [13]. The implications of the explicit form of the solution of the tacnode $\mathrm{RH}$ problem for the tacnode 
process was discussed by Delvaux [10], who made the connection between $[12]$ and the different sets of formulas derived in $[2,3,17,23]$. We briefly discuss it in the section 4.1 .

The critical kernel in the two-matrix model is due to Duits and Geudens [13]. Theorem 2.8 yields an explicit integral representation for this correlation kernel, as stated in Theorem 2.9 and shown in section 4.2.

\subsection{Tacnode kernel}

The tacnode kernel is

$$
K^{t a c}\left(x, y ; r_{1}, r_{2}, s_{1}, s_{2}, \tau\right)=\frac{1}{2 \pi i(x-y)}\left(\begin{array}{llll}
0 & 0 & 1 & 1
\end{array}\right) \widehat{M}^{-1}(y) \widehat{M}(x)\left(\begin{array}{l}
1 \\
1 \\
0 \\
0
\end{array}\right)
$$

where $\widehat{M}$ denotes the analytic continuation of the restriction of $M$ to the region $\Omega_{1}$ around the positive imaginary axis. This was obtained for $\tau=0$ in $[12 \text {, Definition 2.6 }]^{1}$ and for general $\tau \in \mathbb{R}$ in $[10$, section 2.2]. Thus by Theorem 2.8 we have

$$
\widehat{M}=\left(\begin{array}{llll}
m^{(3)} & m^{(0)} & m^{(1)} & m^{(2)}
\end{array}\right),
$$

see also Figure 2 .

Because of the symmetry, see [9, Lemma 5.1] or [10, Lemma 3.1],

$$
M^{-1}(z ; \tau)=\left(\begin{array}{cc}
O & -I \\
I & O
\end{array}\right) M(z ;-\tau)^{T}\left(\begin{array}{cc}
O & I \\
-I & O
\end{array}\right)
$$

(we use $M(z ; \tau)$ to denote the dependence on $\tau$, and $I$ is the $2 \times 2$ identity matrix), we can also write

$$
\begin{aligned}
& K^{t a c}\left(x, y ; r_{1}, r_{2}, s_{1}, s_{2}, \tau\right) \\
& \quad=\frac{1}{2 \pi i(x-y)}\left(\begin{array}{cccc}
1 & 1 & 0 & 0
\end{array}\right) \widehat{M}(y ;-\tau)^{T}\left(\begin{array}{cc}
O & I \\
-I & O
\end{array}\right) \widehat{M}(x ; \tau)\left(\begin{array}{l}
1 \\
1 \\
0 \\
0
\end{array}\right) .
\end{aligned}
$$

\footnotetext{
${ }^{1}$ There is a misprint in formula $(2.47)$ in [12]. It should be $M^{-1}(v) M(u)$ instead of $M^{-1}(u) M(v)$.
} 
Thus $K^{t a c}$ only depends on the sum of the first two columns of $\widehat{M}$. If we put

$$
\widehat{m}=m^{(0)}+m^{(3)}
$$

then we get by (4.2) and (4.4)

$$
\begin{array}{r}
K^{t a c}\left(x, y ; r_{1}, r_{2}, s_{2}, s_{2}, \tau\right)=\frac{1}{2 \pi i(x-y)} \widehat{m}(y ;-\tau)^{T}\left(\begin{array}{cc}
O & I \\
-I & O
\end{array}\right) \widehat{m}(x ; \tau) \\
=\frac{1}{2 \pi i(x-y)}\left(-\widehat{m}_{1}(x ; \tau) \widehat{m}_{3}(y,-\tau)+\widehat{m}_{3}(x ; \tau) \widehat{m}_{1}(y,-\tau)\right. \\
\left.-\widehat{m}_{2}(x ; \tau) \widehat{m}_{4}(y,-\tau)+\widehat{m}_{4}(x ; \tau) \widehat{m}_{2}(y,-\tau)\right) .
\end{array}
$$

A remarkably simple expression for $\left(\frac{\partial}{\partial x}+\frac{\partial}{\partial y}\right) K^{t a c}(x, y)$ can be obtained from (4.1). Using the differential equation (2.7) for $\widehat{M}$ and the formula (2.8) for $U$, we obtain

$$
\begin{aligned}
&\left(\frac{\partial}{\partial x}+\frac{\partial}{\partial y}\right) K^{t a c}(x, y) \\
& \frac{1}{2 \pi i(x-y)}\left(\begin{array}{llll}
0 & 0 & 1 & 1
\end{array}\right) \widehat{M}^{-1}(y)(U(x)-U(y)) \widehat{M}(x)\left(\begin{array}{l}
1 \\
1 \\
0 \\
0
\end{array}\right) \\
&=\frac{1}{2 \pi}\left(\begin{array}{llll}
0 & 0 & 1 & 1
\end{array}\right) \widehat{M}^{-1}(y)\left(r_{1} E_{3,1}-r_{2} E_{4,2}\right) \widehat{M}(x)\left(\begin{array}{l}
1 \\
1 \\
0 \\
0
\end{array}\right)
\end{aligned}
$$

where $E_{j, k}$ is the matrix with 1 in position $j, k$ and 0 otherwise. Combining this with (4.3) we get

$$
\left(\frac{\partial}{\partial x}+\frac{\partial}{\partial y}\right) K^{t a c}(x, y)=\frac{1}{2 \pi}\left(r_{1} \widehat{m}_{1}(y ;-\tau) \widehat{m}_{1}(x ; \tau)-r_{2} \widehat{m}_{2}(y ;-\tau) \widehat{m}_{2}(x ; \tau)\right) \text {. }
$$

where $\widehat{m}=m^{(0)}+m^{(3)}$ as in (4.5).

Delvaux [10] further analyzed (4.6), (4.7) using the formulas (2.29) and (2.32) for $m^{(0)}$ and $m^{(3)}$, and showed that the expression for the tacnode kernel agrees with the one given by Ferrari and Vetö [17]. 


\subsection{Duits-Geudens critical kernel and proof of Theorem 2.9}

We finally prove the Theorem 2.9 about the critical kernel in the two-matrix model obtained by Duits and Geudens in [13].

Proof. We use (4.3) and Theorem 2.8 to rewrite (2.43) as

$$
\begin{aligned}
& K^{c r i t}(x, y ; s, \tau) \\
& \quad=\frac{1}{2 \pi i(x-y)}\left(\begin{array}{cccc}
0 & 0 & 1 & -1) M(i x ;-\tau)^{T}\left(\begin{array}{cc}
O & I \\
-I & O
\end{array}\right) M(i y ; \tau)\left(\begin{array}{l}
1 \\
1 \\
0 \\
0
\end{array}\right) \\
= & \frac{1}{2 \pi i(x-y)}\left(m^{(1)}-m^{(2)}\right)(i x,-\tau)^{T}\left(\begin{array}{cc}
O & I \\
-I & O
\end{array}\right)\left(m^{(0)}+m^{(3)}\right)(i y, \tau) .
\end{array}\right.
\end{aligned}
$$

The critical kernel thus depends on $\widehat{m}=m^{(0)}+m^{(3)}$, see (4.5), which also appeared in the tacnode kernel, and on

$$
\widetilde{m}=m^{(1)}-m^{(2)}=m^{(5)}-m^{(4)} .
$$

The second identity in (4.9) holds because of (2.36).

Another formula for $K^{\text {crit }}$ comes from differentiating (2.43) with respect to $s$. There are differential equations $\frac{\partial M}{\partial s_{1}}=V_{1} M$ and $\frac{\partial M}{\partial s_{2}}=V_{2} M$, which if $s=s_{1}=s_{2}$ as in (2.41) leads to

$$
\frac{\partial M}{\partial s}=V M, \quad V=V_{1}+V_{2},
$$

and $V$ is given explicitly in [9, Proposition 5.11]. This formula implies

$$
V(x)-V(y)=-2 i(x-y)\left(E_{3,1}+E_{4,2}\right),
$$

from which it follows that

$$
\begin{aligned}
\frac{\partial}{\partial s} M^{-1}(i x) M(i y) & =M^{-1}(i x)(V(i y)-V(i x)) M(i y) \\
& =-2(x-y) M^{-1}(i x)\left(E_{3,1}+E_{4,2}\right) M(i y)
\end{aligned}
$$


Thus from (2.43) and (4.3) we get

$$
\begin{aligned}
& \frac{\partial}{\partial s} K^{c r i t}(x, y ; s, \tau) \\
& \quad=\frac{-1}{\pi i}\left(\begin{array}{llll}
-1 & 1 & 0 & 0
\end{array}\right) M(i x)^{-1}\left(E_{3,1}+E_{4,2}\right) M(i y)\left(\begin{array}{l}
1 \\
1 \\
0 \\
0
\end{array}\right) \\
& =\frac{-1}{\pi i}\left(\begin{array}{llll}
0 & 0 & 1 & -1
\end{array}\right) M(i x ;-\tau)^{T}\left(E_{1,1}+E_{2,2}\right) M(i y ; \tau)\left(\begin{array}{l}
1 \\
1 \\
0 \\
0
\end{array}\right),
\end{aligned}
$$

which is somewhat similar to the expression (4.7) for the tacnode kernel, except that the two solutions (4.5) and (4.9) of the ODE (2.13) are now involved. Indeed, by (4.12) and the solution of the tacnode $\mathrm{RH}$ problem

$$
\begin{aligned}
\frac{\partial}{\partial s} K^{c r i t}(x, y ; s, \tau) & =\frac{-1}{\pi i} \widetilde{m}(i x ;-\tau)^{T}\left(E_{11}+E_{22}\right) \widehat{m}(i y ; \tau) \\
& =\frac{-1}{\pi i}\left[\widetilde{m}_{1}(i x ;-\tau) \widehat{m}_{1}(i y ; \tau)+\widetilde{m}_{2}(i x ;-\tau) \widehat{m}_{2}(i y ; \tau)\right],
\end{aligned}
$$

which is a rank two kernel.

Let us check that (4.13) is real-valued. The symmetries of the tacnode $\mathrm{RH}$ problem, see [9, Lemma 5.1],

$$
\begin{array}{rlr}
\overline{M(z)} & =\left(\begin{array}{cc}
I & O \\
O & -I
\end{array}\right) M(\bar{z})\left(\begin{array}{cc}
I & O \\
O & -I
\end{array}\right), \\
M(-z) & =\left(\begin{array}{cc}
J & O \\
O & -J
\end{array}\right) M(z)\left(\begin{array}{cc}
J & O \\
O & -J
\end{array}\right), \quad J=\left(\begin{array}{ll}
0 & 1 \\
1 & 0
\end{array}\right) .
\end{array}
$$

imply for $z=i x$ with $x$ real,

$$
\overline{M(i x)}=\left(\begin{array}{cc}
J & O \\
O & J
\end{array}\right) M(i x)\left(\begin{array}{cc}
J & O \\
O & J
\end{array}\right) .
$$

In view of the solution for $M$ in Theorem 2.8 , this means that for real $x, y$,

$$
\begin{aligned}
& \overline{m_{1}^{(1)}(i x)}=m_{2}^{(2)}(i x), \quad \overline{m_{1}^{(2)}(i x)}=m_{2}^{(2)}(i x), \\
& \overline{m_{1}^{(0)}(i y)}=m_{2}^{(3)}(i y), \quad \overline{m_{1}^{(3)}(i y)}=m_{2}^{(0)}(i y) .
\end{aligned}
$$


Using this and (4.5), (4.9) in (4.13), we find

$$
\frac{\partial}{\partial s} K^{c r i t}(x, y ; s, \tau)=\frac{-2}{\pi} \operatorname{Im}\left[\widetilde{m}_{1}(i x, s,-\tau) \widehat{m}_{1}(i y, s, \tau)\right]
$$

which is real-valued (as it should be).

Since $K^{\text {crit }}(x, y ; s, \tau) \rightarrow 0$ as $s \rightarrow+\infty$, we recover $K^{\text {crit }}$ from (4.14) after integration with respect to $s$, and this leads to (2.44), which is maybe the simplest form for the Duits-Geudens critical kernel.

\section{Appendix: Proof of Lemma 3.2}

Proof. Throughout the proof of Lemma 3.2 we simply write $\infty$ instead of $\infty \omega^{2 k}$, but it is of course understood that the integrals extend to infinity in the appropriate direction.

In addition to the differential identities (3.10)-(3.14) for the functions $Q_{t}, P_{t}$, and $R_{t}$, there are further identities in $[25,26]$ that involve the four functions of the variable $t$ defined by

$$
q(t)=Q_{t}(t), \quad p(t)=P_{t}(t), \quad u(t)=R_{t}(t, t), \quad v(t)=\frac{1}{2}\left(u^{2}-q^{2}\right) .
$$

These four functions satisfy the closed differential system

$$
q^{\prime}=p-u q, \quad p^{\prime}=t q+u q-2 v q, \quad u^{\prime}=-q^{2}, \quad v^{\prime}=-p q .
$$

The second order system (3.2) gives formulas for $\frac{\partial^{2}}{\partial z^{2}} \psi_{1}$ and $\frac{\partial^{2}}{\partial z^{2}} \psi_{2}$. We start by verifying the latter.

From (3.8) and (3.9) we obtain

$$
\begin{aligned}
\frac{\partial^{2}}{\partial z^{2}} \psi_{2}= & -2 r_{2}^{2} \mu \frac{\partial}{\partial z} \psi_{2}+\left(r_{2}^{2} z+2 r_{2} s_{2}-r_{2}^{4} \mu^{2}\right) \psi_{2} \\
& +C r_{2}^{2} \int_{t}^{\infty}(x-t) F(z+C(x-t)) R_{t}(x, t) d x .
\end{aligned}
$$

Here we use (2.20), (3.13) and (5.2) to obtain

$$
(x-t) R_{t}(x, t)=p Q_{t}(x)-q P_{t}(x)=q^{2} R_{t}(x, t)+q^{\prime} Q_{t}(x)-q Q_{t}^{\prime}(x),
$$


where $p=p(t)$. Thus by (3.7) and (3.8),

$$
\begin{aligned}
\frac{\partial^{2}}{\partial z^{2}} \psi_{2} & =-2 r_{2}^{2} \mu \frac{\partial}{\partial z} \psi_{2}+\left(r_{2}^{2} z+2 r_{2} s_{2}-r_{2}^{4} \mu^{2}\right) \psi_{2} \\
& +C r_{2}^{2} \int_{t}^{\infty} F(z+C(x-t))\left(q^{2} R_{t}(x, t)+q^{\prime} Q_{t}(x)-q Q_{t}^{\prime}(x)\right) d x \\
& =-2 r_{2}^{2} \mu \frac{\partial}{\partial z} \psi_{2}+\left(r_{2}^{2} z+2 r_{2} s_{2}-r_{2}^{4} \mu^{2}\right) \psi_{2}+C r_{2}^{2} q^{2}\left(\psi_{2}-F(z)\right) \\
& -C r_{2}^{2} q^{\prime} \psi_{1}-C r_{2}^{2} q \int_{t}^{\infty} F(z+C(x-t)) Q_{t}^{\prime}(x) d x
\end{aligned}
$$

We apply integration by parts to the remaining integral. The integrated term is $C r_{2}^{2} q^{2} F(z)$ and so we obtain (again using (3.7))

$$
\begin{aligned}
\frac{\partial^{2}}{\partial z^{2}} \psi_{2} & =-2 r_{2}^{2} \mu \frac{\partial}{\partial z} \psi_{2}+\left(r_{2}^{2} z+2 r_{2} s_{2}+C r_{2}^{2} q^{2}-r_{2}^{4} \mu^{2}\right) \psi_{2} \\
& -C r_{2}^{2} q^{\prime} \psi_{1}+C^{2} r_{2}^{2} q \int_{t}^{\infty} F^{\prime}(z+C(x-t)) Q_{t}(x) d x \\
& =-2 r_{2}^{2} \mu \frac{\partial}{\partial z} \psi_{2}+\left(r_{2}^{2} z+2 r_{2} s_{2}+C r_{2}^{2} q^{2}-r_{2}^{4} \mu^{2}\right) \psi_{2} \\
& -C^{2} r_{2}^{2} q \frac{\partial}{\partial z} \psi_{1}-C r_{2}^{2} q^{\prime} \psi_{1}
\end{aligned}
$$

which is what is needed for $\frac{\partial^{2}}{\partial z^{2}} \psi_{2}$ according to (3.2).

The proof for $\frac{\partial^{2}}{\partial z^{2}} \psi_{1}$ is a bit more involved. We have by (3.7) and (3.9)

$$
\begin{aligned}
\frac{\partial^{2}}{\partial z^{2}} \psi_{1}= & -\int_{t}^{\infty} F^{\prime \prime}(z+C(x-t)) Q_{t}(x) d x \\
= & -2 r_{2}^{2} \mu \frac{\partial}{\partial z} \psi_{1}+\left(r_{2}^{2} z+2 r_{2} s_{2}-r_{2}^{4} \mu^{2}-C r_{2}^{2} t\right) \psi_{1} \\
& -C r_{2}^{2} \int_{t}^{\infty} x F(z+C(x-t)) Q_{t}(x) d x .
\end{aligned}
$$

Here we use (3.14) and (3.13) to obtain

$$
\begin{aligned}
x Q_{t}(x) & =2 v Q_{t}(x)-p R_{t}(x, t)+P_{t}^{\prime}(x)-u P_{t}(x) \\
& =-\left(u^{2}-2 v\right) Q_{t}(x)-(p-u q) R_{t}(x, t)+P_{t}^{\prime}(x)-u Q_{t}^{\prime}(x),
\end{aligned}
$$

which by (5.2) and the formula for $v(t)$ in (5.1) gives

$$
x Q_{t}(x)=-q^{2} Q_{t}(x)-q^{\prime} R_{t}(x, t)+P_{t}^{\prime}(x)-u Q_{t}^{\prime}(x) .
$$


Using this in (5.3) we find using the definitions (3.7) and (3.8),

$$
\begin{aligned}
\frac{\partial^{2}}{\partial z^{2}} \psi_{1} & =-2 r_{2}^{2} \mu \frac{\partial}{\partial z} \psi_{1}+\left(r_{2}^{2} z+2 r_{2} s_{2}-r_{2}^{4} \mu^{2}-C r_{2}^{2} t-C r_{2}^{2} q^{2}\right) \psi_{1} \\
& +C r_{2}^{2} q^{\prime}\left(\psi_{2}-F(z)\right)-C r_{2}^{2} \int_{t}^{\infty} F(z+C(x-t))\left(P_{t}^{\prime}(x)-u Q_{t}^{\prime}(x)\right) d x .
\end{aligned}
$$

We integrate by parts on the remaining integral. The term $-C r_{2}^{2} q^{\prime} F(z)$ is cancelled by the integrated term $C r_{2}^{2}(p-u q) F(z)$ because of (5.2). Thus

$$
\begin{aligned}
\frac{\partial^{2}}{\partial z^{2}} \psi_{1} & =-2 r_{2}^{2} \mu \frac{\partial}{\partial z} \psi_{1}+\left(r_{2}^{2} z+2 r_{2} s_{2}-r_{2}^{4} \mu^{2}-C r_{2}^{2} t-C r_{2}^{2} q^{2}\right) \psi_{1} \\
& +C r_{2}^{2} q^{\prime} \psi_{2}+C^{2} r_{2}^{2} \int_{t}^{\infty} F^{\prime}(z+C(x-t))\left(P_{t}(x)-u Q_{t}(x)\right) d x
\end{aligned}
$$

Now we use (3.13) again and we simplify using (3.8)

$$
\begin{aligned}
\frac{\partial^{2}}{\partial z^{2}} \psi_{1} & =-2 r_{2}^{2} \mu \frac{\partial}{\partial z} \psi_{1}+\left(r_{2}^{2} z+2 r_{2} s_{2}-r_{2}^{4} \mu^{2}-C r_{2}^{2} t-C r_{2}^{2} q^{2}\right) \psi_{1} \\
& +C r_{2}^{2} q^{\prime} \psi_{2}-C^{2} r_{2}^{2} \int_{t}^{\infty} F^{\prime}(z+C(x-t))\left(q R_{t}(x, t)-Q_{t}^{\prime}(x)\right) d x \\
& =-2 r_{2}^{2} \mu \frac{\partial}{\partial z} \psi_{1}+\left(r_{2}^{2} z+2 r_{2} s_{2}-r_{2}^{4} \mu^{2}-C r_{2}^{2} t-C r_{2}^{2} q^{2}\right) \psi_{1} \\
& +C r_{2}^{2} q^{\prime} \psi_{2}-C^{2} r_{2}^{2} q\left(\frac{\partial}{\partial z} \psi_{2}-F^{\prime}(z)\right)+C^{2} r_{2}^{2} \int_{t}^{\infty} F^{\prime}(z+C(x-t)) Q_{t}^{\prime}(x) d x
\end{aligned}
$$

We integrate by parts, the integrated term cancels with $C^{2} r_{2}^{2} q F^{\prime}(z)$ and so

$$
\begin{aligned}
\frac{\partial^{2}}{\partial z^{2}} \psi_{1} & =-2 r_{2}^{2} \mu \frac{\partial}{\partial z} \psi_{1}+\left(r_{2}^{2} z+2 r_{2} s_{2}-r_{2}^{4} \mu^{2}-C r_{2}^{2} t-C r_{2}^{2} q^{2}\right) \psi_{1} \\
& -C^{2} r_{2}^{2} q \frac{\partial}{\partial z} \psi_{2}+C r_{2}^{2} q^{\prime} \psi_{2}-C^{3} r_{2}^{2} \int_{t}^{\infty} F^{\prime \prime}(z+C(x-t)) Q_{t}(x) d x .
\end{aligned}
$$

The final integral we can express in terms of $\frac{\partial^{2}}{\partial z^{2}} \psi_{1}$ (see the first identity in (5.3)) and the result is that

$$
\begin{aligned}
\frac{\partial^{2}}{\partial z^{2}} \psi_{1} & =-2 r_{2}^{2} \mu \frac{\partial}{\partial z} \psi_{1}+\left(r_{2}^{2} z+2 r_{2} s_{2}-r_{2}^{4} \mu^{2}-C r_{2}^{2} t-C r_{2}^{2} q^{2}\right) \psi_{1} \\
& -C^{2} r_{2}^{2} q \frac{\partial}{\partial z} \psi_{2}+C r_{2}^{2} q^{\prime} \psi_{2}+C^{3} r_{2}^{2} \frac{\partial^{2}}{\partial z^{2}} \psi_{1} .
\end{aligned}
$$

Rearranging terms we find

$$
\begin{aligned}
\left(C^{3} r_{2}^{2}-1\right) \frac{\partial^{2}}{\partial z^{2}} \psi_{1} & =+C^{2} r_{2}^{2} q \frac{\partial}{\partial z} \psi_{2}+2 r_{2}^{2} \mu \frac{\partial}{\partial z} \psi_{1} \\
& -C r_{2}^{2} q^{\prime} \psi_{2}+\left(-r_{2}^{2} z-2 r_{2} s_{2}+r_{2}^{4} \mu^{2}+C r_{2}^{2} t+C r_{2}^{2} q^{2}\right) \psi_{1}
\end{aligned}
$$


Since $C^{3} r_{2}^{2}-1=\left(r_{2} / r_{1}\right)^{2}$, this is

$$
\begin{aligned}
\frac{\partial^{2}}{\partial z^{2}} \psi_{1} & =C^{2} r_{1}^{2} q \frac{\partial}{\partial z} \psi_{2}+2 r_{1}^{2} \mu \frac{\partial}{\partial z} \psi_{1} \\
& -C r_{1}^{2} q^{\prime} \psi_{2}+\left(-r_{1}^{2} z-2 \frac{r_{1}^{2}}{r_{2}} s_{2}+r_{1}^{2} r_{2}^{2} \mu^{2}+C r_{1}^{2} t+C r_{1}^{2} q^{2}\right) \psi_{1}
\end{aligned}
$$

Since $t=C^{-1}\left(2 \frac{s_{1}}{r_{1}}+2 \frac{s_{2}}{r_{2}}-\left(r_{1}^{2}+r_{2}^{2}\right) \mu^{2}\right)$, we finally obtain

$$
\begin{aligned}
\frac{\partial^{2}}{\partial z^{2}} \psi_{1} & =C^{2} r_{1}^{2} q \frac{\partial}{\partial z} \psi_{2}+2 r_{1}^{2} \mu \frac{\partial}{\partial z} \psi_{1} \\
& -C r_{1}^{2} q^{\prime} \psi_{2}+\left(-r_{1}^{2} z+2 r_{1} s_{1}-r_{1}^{4} \mu^{2}+C r_{1}^{2} q^{2}\right) \psi_{1}
\end{aligned}
$$

as claimed in (3.2).

Acknowledgements I am grateful to Steven Delvaux and Dries Geudens for useful discussions about the tacnode problem and the critical kernels.

The author is supported by KU Leuven Research Grant No. OT/12/073, the Belgian Interuniversity Attraction Pole P07/18, FWO Flanders projects G.0641.11 and G.0934.13, and by Grant No. MTM2011-28952-C02 of the Spanish Ministry of Science and Innovation.

\section{References}

[1] M. Adler, S. Chhita, K. Johansson, and P. van Moerbeke, Tacnode GUE-minor processes and double Aztec diamonds, preprint arXiv: 1303.5279.

[2] M. Adler, P.L. Ferrari, and P. van Moerbeke, Non-intersecting random walks in the neighborhood of a symmetric tacnode, Ann. Probab. 41 (2013), 2599-2647.

[3] M. Adler, K. Johansson, and P. van Moerbeke, Double Aztec diamonds and the tacnode process, preprint arXiv:1112.5532.

[4] G. Anderson, A. Guionnet, and O. Zeitouni, Cambridge University Press, Cambridge, 2010.

[5] J. Baik, Painlevé formulas of the limiting distributions for non-null complex sample covariance matrices, Duke Math. J. 133 (2006), 205235. 
[6] J. Baik, K. Liechty, and G. Schehr, On the joint distribution of the maximum and its position of the Airy 2 process minus a parabola, J. Math. Phys. 53 (2012), 083303.

[7] M. Bertola and M. Cafasso, The gap probabilities of the tacnode, Pearcey and Airy point processes, their mutual relationship and evaluation, preprint arXiv:1303.2894.

[8] P. Deift, Integrable operators, in: Differential operators and spectral theory (V. Buslaev et al., eds). Amer. Math. Soc., Providence, RI, 1999, pp. 69-84.

[9] S. Delvaux, Non-intersecting squared Bessel paths at a hard-edge tacnode, preprint arXiv:1204.4430, to appear in Comm. Math. Phys.

[10] S. Delvaux, The tacnode kernel: equality of Riemann-Hilbert and Airy resolvent formulas, preprint arXiv:1211.4845.

[11] S. Delvaux, D. Geudens, and L. Zhang, Universality and critical behavior in the chiral two-matrix model, Nonlinearity 26 (2013) 2231-2298.

[12] S. Delvaux, A.B.J. Kuijlaars and L. Zhang, Critical behavior of nonintersecting Brownian motions at a tacnode, Comm. Pure Appl. Math. 64 (2011), 1305-1383.

[13] M. Duits and D. Geudens, A critical phenomenon in the two-matrix model in the quartic/quadratic case, Duke Math. J. 162 (2013), 13831462 .

[14] M. Duits, D. Geudens, and A.B.J. Kuijlaars, A vector equilibrium problem for the two-matrix model in the quartic/quadratic case, Nonlinearity 24 (2011), 951-993.

[15] M. Duits, A.B.J. Kuijlaars, and M.Y. Mo, The Hermitian two matrix model with an even quartic potential, Mem. Amer. Math. Soc. 217 (2012), no. 1022, 105 pp.

[16] B. Eynard and M.L. Mehta, Matrices coupled in a chain I. Eigenvalue correlations, J. Phys. A. 31 (1998), 4449-4456.

[17] P. Ferrari and B. Vetö, Non-colliding Brownian bridges and the asymmetric tacnode process, Electron. J. Probab. 17 (2012), 1-17.

[18] H. Flaschka and A.C. Newell, Monodromy and spectrum-preserving deformations I, Comm. Math. Phys. 76 (1980), 65-116. 
[19] A.S. Fokas, A.R. Its, A.A. Kapaev, and V. Yu. Novokshenov, Painlevé Transcendents. The Riemann-Hilbert Approach. Amer. Math. Soc., Providence, RI, 2006.

[20] D. Geudens and L. Zhang, Transitions between critical kernels: from the tacnode kernel and critical kernel in the two-matrix model to the Pearcey kernel, preprint arXiv:1208.0762.

[21] S.P. Hastings and J.B. McLeod, A boundary value problem associated with the second Painlevé transcendent and the Korteweg-de Vries equation, Arch. Rational Mech. Anal. 73 (1980), 31-51.

[22] A.R. Its, A.G. Izergin, V.E. Korepin, and N.A. Slavnov, Differential equations for quantum correlation functions, Intern. J. Mod. Phys. B4 (1990), 1003-1037.

[23] K. Johansson, Non-colliding Brownian motions and the extended tacnode process, Comm. Math. Phys. 319 (2013), 231-267.

[24] P.D. Miller, Applied Asymptotic Analysis, Amer. Math. Soc., Providence RI, 2006.

[25] C. Tracy and H. Widom, Level-spacing distributions and the Airy kernel, Comm. Math. Phys. 159 (1994), 151-174.

[26] C.A. Tracy and H. Widom, Airy kernel and Painlevé II, in: Isomonodromic deformations and applications in physics (J. Harnad and A. Its, eds.), Amer. Math. Soc., Providence, RI, 2002 pp. 85-96. 